\title{
GaN Resonant Cavity Light-Emitting Diodes for Plastic Optical Fiber Applications
}

\author{
A. J. Shaw, A. L. Bradley, J. F. Donegan, and J. G. Lunney
}

\begin{abstract}
The optical designs of resonant GaN light-emitting diodes (LEDs) have been determined for maximum extraction efficiency into typical plastic optical fiber of numerical aperture 0.5. An optimum extraction efficiency of $3.9 \%$ can be achieved for a practical resonant cavity LED (RCLED), taking account of current growth and processing considerations. The optimized device is a metal-active layer distributed Bragg reflector construction. Constructive interference effects from the top metal mirror are found to play the dominant role in efficiency enhancement. The extraction efficiency of an optimized resonant single-mirror LED is found to be $3.3 \%$, indicating a small compromise in performance compared with the more complex RCLED structure.
\end{abstract}

Index Terms-High-temperature applications, light-emitting diode (LED), plastic optical fiber (POF), resonant cavity enhancement.

\section{INTRODUCTION}

$\mathbf{R}$ ESONANT cavity light-emitting diodes (RCLEDs) have the capacity to produce higher spectral purity and increased directionality of emission compared to conventional light-emitting diodes (LEDs) [1]. RCLEDs have shown considerable promise as sources for plastic optical fiber (POF)-based networks, where the large core diameter of POF and relatively low bandwidth $(<1 \mathrm{GHz})$ requirements are compatible with current RCLED technology. Red AlGaInP-based RCLEDs, matching the 650-nm POF transmission window, are ready for large-scale production [2]. Additional higher transmission windows, at 510 and $570 \mathrm{~nm}$, are accessible using III-V nitride semiconductors which, with less temperature dependent properties than AlGaInP, are more suitable for automotive and avionic environments. Blue, violet, and green GaN RCLEDs have been demonstrated showing clear cavity effects in the emission spectrum and directionality of emission [3]-[5], though no effort was made to optimize the cavity design for maximum light extraction efficiency.

We report on the optical design of GaN resonant LEDs emitting at $510 \mathrm{~nm}$ into a typical POF numerical aperture (NA) of 0.5. Key design parameters are discussed and performances of resonant cavity and resonant single-mirror devices are compared. The model is described elsewhere [6], [7]. The refractive index values for $\mathrm{Al}_{x} \mathrm{Ga}_{1-x} \mathrm{~N}, \mathrm{In}_{y} \mathrm{Ga}_{1-y} \mathrm{~N}$, and the metal top mirror were taken from the literature [8]-[10]. All calculations

Manuscript received March 10, 2004; revised May 26, 2004. Financial support was provided by the IST ESPRIT 1999-10292 “AGETHA" Project.

The authors are with the Physics Department, Trinity College, Dublin 2, Ireland (e-mail: ajshaw@gemini.tcd.ie; bradlel@tcd.ie; jdonegan@tcd.ie; jlunney@tcd.ie).

Digital Object Identifier 10.1109/LPT.2004.833116 assume an intrinsic quantum-well (QW) emission spectrum centered on $510 \mathrm{~nm}$ with a full-width at half-maximum of $30 \mathrm{~nm}$.

\section{RCLED DEVICES}

Essentially three mirror types can be utilized to form the cavity: metallic, epitaxially grown $\mathrm{AlGaN}-\mathrm{GaN}$ distributed Bragg reflector (DBR), and dioxide-based DBR (e.g., $\mathrm{SiO}_{2}-\mathrm{TiO}_{2}$ ). Both metallic and $\mathrm{SiO}_{2}-\mathrm{TiO}_{2}$ DBR bottom mirrors require difficult and expensive liftoff processes, rendering an $\mathrm{AlGaN}-\mathrm{GaN}$ DBR the preferable choice. With transparent substrates and high reflectivity metallic or $\mathrm{SiO}_{2}-\mathrm{TiO}_{2}$ DBR mirrors available for the back (top) mirror, a substrate emitting RCLED structure is the logical choice. $\mathrm{SiO}_{2}-\mathrm{TiO}_{2}$ is the preferred option, exhibiting the highest reflectivity $(R>99 \%$ for five pairs) and a broad angular-spectral reflectivity stopband. However, if good electrical characteristics, including uniform current injection and low operating voltage, are to be achieved, an additional metallic layer has to be included under the insulating $\mathrm{SiO}_{2}-\mathrm{TiO}_{2} \mathrm{DBR}$. Absorption resulting from this layer reduces the performance of the dioxide DBR cavity structure to less than what can be achieved using a top metal mirror. A good choice for the metallic top mirror is a combination of a thin palladium (Pd) layer, which ensures good electrical contact, and a thicker silver $(\mathrm{Ag})$ layer that produces a reflectivity of $>70 \%$ over all angles across the required spectral range [10].

Ideally, the bottom DBR would consist of five alternate $\lambda / 4$ AlN-GaN pair layers. Despite progress [11], [12], current epitaxial growth considerations limit the DBR Al fractions to between 0.25 and 0.4 [13], [14]. Therefore, a realistic middle value of 0.3 is considered. The relatively small refractive index variation of $\mathrm{Al}_{x} \mathrm{Ga}_{1-x} \mathrm{~N}$ with $\mathrm{Al}$ fraction impacts on the spectral-angular stopband of the mirror and increases the effective length due to the penetration depth into the DBR, having important implications for the design of these devices. Furthermore, in order to avoid having to dope the AlGaN-GaN DBR, intracavity contacts are required, resulting in an increase in the overall cavity length.

Three design parameters are simultaneously optimized: the number of layer pairs in the DBR, the cavity thickness, and the position of the quantum wells. The active region consists of three 2-nm InGaN QWs and 13-nm GaN barriers. An ideal $1 \lambda$ RCLED structure, incorporating a five-pair layer $\mathrm{SiO}_{2}-\mathrm{TiO}_{2}$ DBR top mirror and a five-pair layer AlN-GaN DBR bottom mirror, yields a calculated extraction efficiency of $8.5 \%$ into an emission NA of 0.5 , compared to $1 \%$ for a bulk GaN LED emitting through a single facet into an emission NA of 0.5. The optimized practical device (Fig. 1) has the same multiple quantum-well (MQW) active region as the ideal RCLED and 


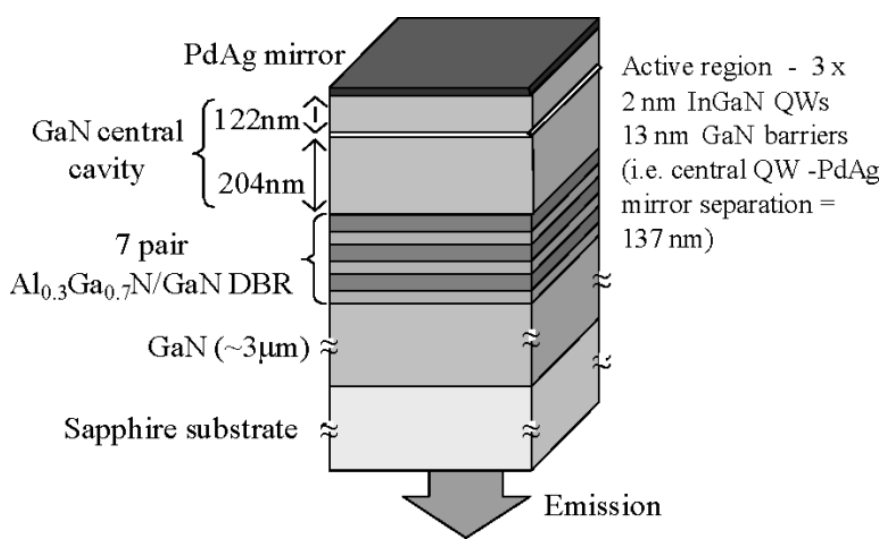

Fig. 1. Optimum optical structure of a GaN RCLED emitting at $510 \mathrm{~nm}$, compatible with electrical and growth considerations.

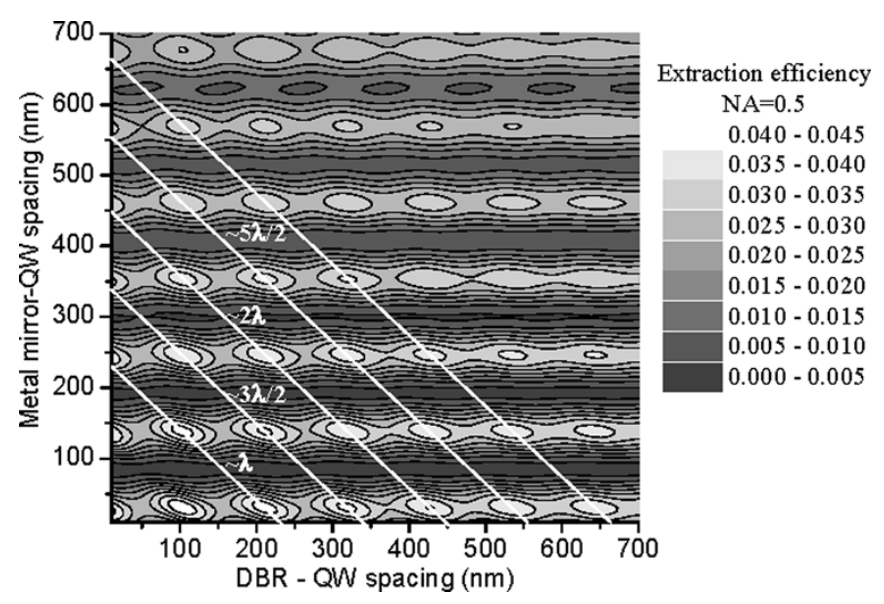

Fig. 2. Contour plot of modeled extraction efficiency into an NA $=0.5$ for a metal- $\mathrm{Al}_{0.3} \mathrm{Ga}_{0.7} \mathrm{~N}-\mathrm{GaN}$ DBR RCLED as functions of top and bottom cavity spacer layers, assuming a QW emission linewidth of $30 \mathrm{~nm}$. The diagonal lines represent structures with constant cavity thickness and, hence, resonant wavelength, with variations along a single diagonal caused by changes in the position of the active region in the cavity. The maxima (minima) along the diagonal correspond to the QW positioned at an antinode (node) of the cavity. The nearer the antinode at which the QW is placed is to the metal mirror, the higher the efficiency.

an $\mathrm{Al}_{0.3} \mathrm{Ga}_{o .7} \mathrm{~N}-\mathrm{GaN}$ DRB. However, the optical central cavity thickness has been increased to $3 \lambda / 2$, allowing for a thicker bottom cavity spacer layer as required for intracavity contact processing. It should also be noted that positioning the MQW close to the metal mirror, at the first antinode, results in the loss of the electroluminescence, attributed to diffusion of the metal into the MQW region [15]. Therefore, in a practical structure, the QWs must be placed at the second antinode from the mirror. The calculated extraction efficiency of this device, into an NA of 0.5 , is $3.9 \%$, assuming the same intrinsic QW emission spectrum as before. This new structure has the benefit of the bottom mirror being compatible with epitaxial growth techniques, while the top metal mirror can act as an electrical contact.

\section{SiNGLE-MIRRor DEVICES}

The importance of the position of the emitter in the cavity can be seen in Fig. 2. As expected, the extraction efficiency exhibits a periodic dependence on the thickness of both cavity spacer layers. However, the variation in efficiency is greatest
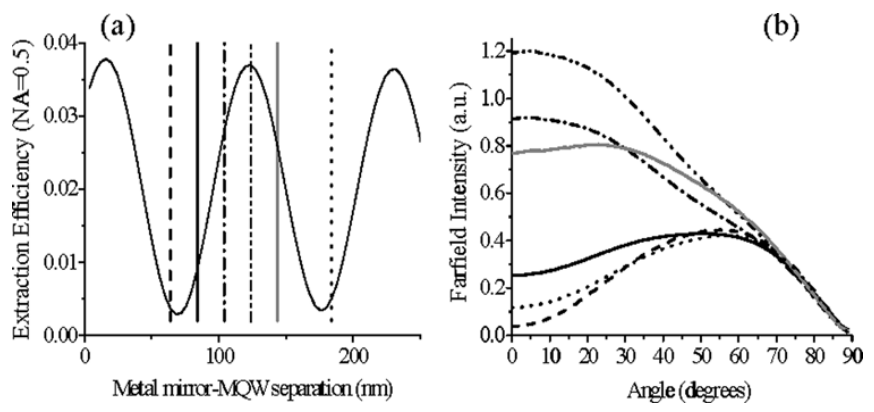

Fig. 3. (a) Extraction efficiency for a $5 \lambda / 4$ RCLED as a function of QW position inside the cavity (with the cavity length fixed at optimum value for maximum efficiency and a bottom DBR with a five-period $\mathrm{Al}_{0.3} \mathrm{Ga}_{0.7} \mathrm{~N}-\mathrm{GaN}$ structure). (b) The simulated farfields for metal mirror-active region separations are indicated by the coded vertical lines in graph (a).

when moving vertically along the contour plot, corresponding to varying the metal-QW spacing while maintaining a constant DBR-QW spacing. Clearly, correct positioning of the QW with respect to the metal mirror is crucial to the performance of the RCLED and it is necessary to have a characterization method for determining this important device parameter. Standard white light reflectivity measurements while providing information on many cavity properties yield no information on the QW position. Modeling studies predict a strong dependence of the shape of the farfield emission profile with QW position, as shown in Fig. 3. When the QW is optimally positioned at an antinode of the cavity, the farfield emission pattern is approximately constant over the $0^{\circ}-25^{\circ}$ angular range, and decreases at greater angles. In contrast, the maximum in the farfield emission pattern occurs at an angle of $50^{\circ}$ from the normal when the QW is positioned at a node of the cavity.

Furthermore, the optimal position for the QW, the first antinode from the metal mirror, is independent of the cavity thickness, as seen in Fig. 2. This suggests that constructive interference effects from the top mirror play a dominant role in the efficiency enhancement produced by this RCLED device. Therefore, the performance of the optimum single-mirror LED was calculated for comparison. The absence of any DBR is a major attraction from a growth perspective. Even though the separation between the metal mirror and the QWs is the only variable in the design process, an optical model similar to that used for the RCLED designs is required in order to take account of the phase shift on reflection at the metal mirror. The calculated extraction efficiency of this device, for the QWs positioned at the second antinode from the metallic mirror, is $3.3 \%$ into an emission NA of 0.5 . Assuming $25 \%$ internal quantum efficiency, this corresponds to $200 \mu \mathrm{W}$ of power into an NA of 0.5 at a bias current of $10 \mathrm{~mA}$, satisfactory for many application requirements.

\section{CONCLUSION}

The optical design of practical GaN RCLEDs has been considered. The position of the QWs within the cavity is seen to be a critical parameter for determining the extraction efficiency. Modeling studies have shown a strong variation in the farfield emission pattern with QW position, thus yielding a practical means for measuring this important parameter. Furthermore, 
due to the low refractive index contrast in the $\mathrm{AlGaN}-\mathrm{GaN}$ DBRs, the performance of the simpler single-mirror resonant device is only marginally less efficient than the more complex RCLED structure.

\section{REFERENCES}

[1] E. F. Schubert, Y.-H. Wang, A. Y. Cho, L.-W. Tu, and G. J. Zydzik, "Resonant cavity light emitting diode," Appl. Phys. Lett., vol. 60, pp. 921-923, 1992.

[2] R. Wirth, C. Karnutsch, S. Kugler, and K. Streubel, "High-efficiency resonant-cavity LEDs emitting at $650 \mathrm{~nm}$," IEEE Photon. Technol. Lett., vol. 13, pp. 421-423, May 2001.

[3] Y.-K. Song, M. Diagne, H. Zhou, A. V. Nurmikko, R. P. Schneider Jr., and T. Takeuchi, "Resonant cavity InGaN quantum well blue light-emitting diodes," Appl. Phys. Lett., vol. 77, pp. 1744-1746, 2000.

[4] M. Diagne, Y. He, H. Zhou, E. Makarona, A. V. Nurmikko, J. Han, T. Takeuchi, and M. Krames, "A high injection resonant cavity violet light emitting diode incorporating (Al,Ga)N distributed Bragg reflector," Physica Status Solidi A, vol. 188, pp. 105-108, 2001.

[5] F. B. Naranjo, S. Fernandez, M. A. Sanchez-Garcia, F. Calle, and E. Calleja, "Resonant-cavity InGaN multiple-quantum-well green light-emitting diode grown by molecular-beam epitaxy," Appl. Phys. Lett., vol. 80, pp. 2198-2200, 2002.

[6] H. Benisty, H. de Neve, and C. Weisbuch, "Impact of planar microcavity effects on light extraction- Part I: Basic concepts and analytical trends," IEEE J. Quantum Electron., vol. 34, pp. 1612-1631, Sept. 1998.
[7] - "Impact of planar microcavity effects on light extraction- Part II: Selected exact simulations and role of photon recycling," IEEE J. Quantum Electron., vol. 34, pp. 1632-1643, Sept. 1998.

[8] G. M. Laws, E. C. Larkins, I. Harrison, C. Molloy, and D. Somerford, "Improved refractive index formulas for $\mathrm{Al}_{x} \mathrm{Ga}_{1-x} \mathrm{~N}$ and $\operatorname{In}_{y} \mathrm{Ga}_{1-y} \mathrm{~N}$ alloys," J. Appl. Phys., vol. 89, pp. 1108-1115, 2001.

[9] M. M. Y. Leung, A. B. Djurisiić, and E. H. Li, "Refractive index of InGaN quantum well," J. Appl. Phys., vol. 84, pp. 6312-6317, 1998.

[10] P. Maaskant, M. Akhter, B. Roycroft, E. O'Carroll, and B. Corbett, "Fabrication of Ga-N based resonant cavity LEDs," Physica Status Solidi A, vol. 192, no. 2, pp. 348-353, 2002.

[11] S. Fernandez, F. B. Naranjo, M. A. Sanchez-Garcia, and E. Calleja, "High-quality distributed Bragg reflectors based on $\mathrm{Al}_{x} \mathrm{Ga}_{1-x} \mathrm{~N} / \mathrm{GaN}$ multilayers grown by molecular-beam epitaxy," Appl. Phys. Lett., vol. 79, pp. 2136-2138, 2001.

[12] H. M. Ng, T. D. Moustakas, and S. N. G. Chu, "High reflectivity and broad bandwidth AlN/GaN distributed Bragg reflectors grown by molecular beam epitaxy," Appl. Phys. Lett., vol. 76, pp. 2818-2820, 2000.

[13] T. Someya and Y. Arakawa, "Highly reflective $\mathrm{GaN} / \mathrm{Al}_{0.34} \mathrm{Ga}_{0.66} \mathrm{~N}$ quarter-wave reflectors grown by metal organic chemical vapou deposition," Appl. Phys. Lett., vol. 73, pp. 3653-3655, 1998.

[14] N. Nakada, M. Nakaji, H. Ishikawa, T. Egawa, M. Umeno, and T. Jimbo, "Improved characteristics of InGaN multiple-quantum-well light-emitting diode by GaN/AlGaN distributed Bragg reflector grown on sapphire," Appl. Phys. Lett., vol. 76, pp. 1804-1806, 2000.

[15] B. Roycroft, M. Akhter, P. Maaskant, B. Corbett, A. Shaw, L. Bradley, P. de Mierry, and M. Poisson, "Origin of power fluctuations in GaN resonant-cavity light-emitting diodes," Opt. Express, vol. 12, pp. 736-741, 2004. 